Itinéraires Itinéraires

Littérature, textes, cultures

2012-3 | 2013

Lire les villes marocaines

\title{
Au regard du sacré : lectures croisées de la médina de Fès
}

Brice Gruet

\section{(2) OpenEdition}

Journals

Édition électronique

URL : http://journals.openedition.org/itineraires/1014

DOI : 10.4000/itineraires.1014

ISSN : 2427-920X

Éditeur

Pléiade

\section{Édition imprimée}

Date de publication : 1 juillet 2013

Pagination : 153-167

ISBN : 978-2-343-01183-7

ISSN : 2100-1340

\section{Référence électronique}

Brice Gruet, «Au regard du sacré : lectures croisées de la médina de Fès », Itinéraires [En ligne], 2012-3 | 2013, mis en ligne le 01 décembre 2012, consulté le 19 avril 2019. URL : http://

journals.openedition.org/itineraires/1014 ; DOI : 10.4000/itineraires.1014

\section{(ब) $(\Theta \Theta$}

Itinéraires est mis à disposition selon les termes de la licence Creative Commons Attribution - Pas d'Utilisation Commerciale - Pas de Modification 4.0 International. 


\title{
Au regard du sacré ${ }^{1}$ : lectures croisées de la médina de Fès
}

\begin{abstract}
This paper focuses on the analysis of Fes medina, Morocco, through the work of Titus Burckhardt, Fes, city of Islam, published in the 1960s. The author emphasizes on the sacred aspects of the city and proposes a thoughtful investigation of the specific history of this urban space. Burckhardt provides us with a critical insight into Fes' evolution within both a colonial and then postcolonial context. He praises the strength of muslim tradition, and especially the sufi one. This vision is confronted with nowadays Fes, the possibilities of development of the whole city and the contradictions of such a project.
\end{abstract}

Keywords : Fes, Islam, sacred, tradition, Burckhardt, cultural heritage Mots clés : Fès, islam, sacré, tradition, Burckhardt, patrimoine

1. Je reprends la définition du sacré donnée par Maurice Godelier dans son ouvrage $A u$ fondement des sociétés humaines: ce que nous apprend l'anthropologie (Paris, Albin Michel, 2007) : "Le sacré et la dimension politique-religieuse de l'imaginaire ont une place décisive. Les représentations et croyances imaginaires constituent des vérités existentielles qui s'incarnent dans des pratiques symboliques. Interprétée en langage occidental, cette institution que l'on appelle initiation dans les sociétés dites primitives est un processus par lequel tous les individus se retrouvent dépendants les uns des autres, pris dans des liens pour nous largement imaginaires : il concerne tous les villages, tous les lignages, tous les individus. Ce qui les unit à ce moment sont des rapports que nous qualifions de politicoreligieux. » Mais cet article s'insérant dans un projet de recherche centré sur une " géographie du sacré », je prends cette définition comme provisoire. 
Je propose dans ce court essai ${ }^{2}$ une évocation de la ville de Fès à travers l'ouvrage de Titus Burckhardt, Fès, ville d'islam ${ }^{3}$, et mon propre séjour effectué sur place au printemps 2008.

C'est seulement après mon retour de Fès que j'ai découvert ce livre, et c'est ce qui m'a donné l'idée d'en reprendre le contenu en le confrontant à la ville actuelle. En effet, il me semblait que l'ouvrage, pourtant écrit dans les années 1960, conservait toute sa pertinence, surtout si l'on sait le rôle que l'auteur a joué dans la préservation de la médina de Fès auprès des autorités marocaines et de l'UNESCO.

Mon travail de géographe procède d'un regard bien particulier, attentif à interpréter un espace urbain dans toutes ses dimensions et toutes ses significations ${ }^{4}$. En ce cas, la « lecture » sensible de l'espace urbain représente bien une métaphore vive et un fil directeur important de ma démarche. En prenant effectivement la ville comme un texte, c'est-à-dire un tissage, celleci autorise alors autant de parcours, autant de lectures en archipel, autant d'interprétations de cet espace par essence composite et en mouvement ${ }^{5}$. C'est de cet entrelacs que je voudrais rendre compte ici.

Il est bien évident que l'aspect empirique et foncièrement personnel de cette approche peut sembler non scientifique. Pourtant, j'ai eu à cœur, depuis plus de dix ans, de développer une méthode qualitative d'analyse des espaces urbains, qui a déjà porté ses fruits, en privilégiant une vision interne, comme j'ai pu le faire avec Rome. À cet égard, le recours à la première personne (plus courante dans le monde universitaire anglo-saxon) est à mon sens plus un gage d'honnêteté intellectuelle qu'un aveu de paresse méthodologique.

2. Ce mot est choisi à escient, il renvoie à ce qui a été défini en géographie comme l'approche «essayiste » par Joël Bonnemaison. Cf. Joël Bonnemaison, Gens de pirogue et gens de la terre. Les fondements géographiques d'une identité, livre I, préface, Paris, ORSTOM, 1996 (réédition remaniée de sa thèse L'Arbre et la Pirogue). La géographie essayiste assume et intègre la part de subjectivité du chercheur dans son propre projet de travail, un peu à l'instar de l'observation participante de B. Malinowski.

3. Titus Burckhardt, Fes, Stadt des Islam, Olten, Urs Graf, 1960. Trad. française de Genia Catala publiée chez Archè à Milan en 2007. C'est à cette édition que je renvoie dans le présent article.

4. En effet, dans la lignée des travaux d'Augustin Berque sur les milieux humains (notamment Écoumène, Paris, Belin, 2000), il me semble préférable d'insister sur le balancement incessant entre intériorité et extériorité (résumé par Berque en termes de médiance et de trajection) plutôt que de parler d'objectif et de subjectif. Depuis Écoumène, Berque a d'ailleurs proposé le terme de mésologie pour prendre en compte cette nouvelle approche.

5. J'ai eu l'occasion d'aborder ces aspects plus en profondeur dans ma thèse de doctorat : La Rue à Rome, miroir de la ville. Entre l'émotion et la norme, Paris, Presses de l'Université Paris-Sorbonne, 2006. J'y renvoie le lecteur pour les aspects méthodologiques. Kevin Lynch, mais aussi Spiro Kostof, Edward T. Hall et, bien sûr, Françoise Choay, ont inspiré mon travail. On peut aussi évoquer Pierre Sansot, avec sa Poétique de la ville (Paris, Klincksieck, 1973). 
Il faut ajouter que la découverte d'un nouvel espace urbain engendre des émotions qui font partie intégrante de la démarche du chercheur : les mettre de côté me semblerait une forme de partialité difficilement défendable $^{6}$. J'assume donc totalement le caractère « subjectif » de cette approche (même si je n'accepte pas vraiment le terme), elle-même basée sur une expérience de la ville à la fois ancienne et intellectuellement fondée. Le texte qui suit est écrit en accord avec la méthode du marcheur qui épouse les moments d'une lecture-promenade, à la manière de Régine Robin, pour qui la ville « n'est pas un objet mais une pratique, un mode d'être, un rythme, une respiration, une poétique ${ }^{7} \gg$. Le travail de cette historienne, sociologue et écrivaine s'inscrit d'ailleurs, pour ses flâneries berlinoises, dans la suite de Walter Benjamin. Je propose de lire à mon tour la ville de Fès en gardant à l'esprit les œuvres de ces deux auteurs.

Titus Burckhardt (1908-1984) n'est pas très connu du monde universitaire français. Pourtant, son œuvre singulière, située au croisement d'une authentique quête spirituelle et de toute la rigueur des sciences humaines, est une œuvre capitale pour approcher la culture musulmane. Burckhardt, citoyen suisse, a connu le Maroc à l'époque du protectorat français puis à son indépendance ${ }^{8}$. C'est dans cette perspective originale que son ouvrage sur Fès prend toute son importance. En effet, Titus Burckhardt a travaillé sa vie durant à la mise en valeur de la vieille ville de Fès, l'une des plus grandes, voire la plus grande, médina du monde musulman. Ce travail a finalement valu à Fès d'être répertoriée dans la liste du Patrimoine mondial de l'humanité de l'UNESCO. Burckhardt est donc un fin connaisseur de la ville. Il appartient aussi, comme Seyyed Hossein Nasr ou Frithjof Schuon, au courant dit pérennialiste, courant attentif à la problématique de la modernité et de la sécularisation des sociétés actuelles qui contraste avec le rapport au sacré des sociétés traditionnelles considéré comme fondateur de leur fonctionnement ${ }^{9}$.

6. Voir, à cet égard, le travail canonique de Maurice Halbwachs dans son édition complète, La Mémoire collective, édition critique de Gérard Namer, Paris, Albin Michel, 1997.

7. Régine Robin, à propos de Megapolis - les derniers pas du flâneur (Paris, Stock, 2009). « Régine Robin », www.franceculture.fr, $1^{\text {er }}$ février 2009.

8. Il a effectué plusieurs longs séjours dans les années 1930, et son dernier voyage est survenu en 1981. Son but, lors de son premier séjour, était de trouver un maître spirituel, en l'occurrence dans le soufisme. Il ne s'agissait donc absolument pas d'accumuler un savoir livresque sur la religion musulmane, mais de la vivre par son versant initiatique. C'est en ce sens qu'il faut comprendre la «spiritualité » envisagée par Burkchardt. La nuance est fondamentale.

9. On peut considérer que ce courant a été fondé par René Guénon (1886-1951), dont les œuvres principales sont partiellement disponibles chez Gallimard, dans la collection « Tradition », créée par Jean Paulhan. Ses études portent sur les symboles et les différentes traditions spirituelles de l'humanité. À cet égard, il est l'un des premiers à mettre en œuvre ce que l'on pourrait appeler une démarche comparatiste dans les sciences humaines, même si son but n'est pas à proprement parler scientifique. En revanche, il va exercer une forte influence sur 
C'est pourquoi le propos de Burckhardt s'inscrit en faux par rapport aux discours sur la modernité, urbaine ou autre. Son point de vue n'est pas celui d'un Européen venant naïvement découvrir de l'extérieur la vie et les us et coutumes d'un pays exotique. Au contraire, son point de vue est intérieur, dans tous les sens du terme, et son point de départ n'est pas la modernité, mais la tradition ${ }^{10}$. Mais commençons l'exploration de la ville actuelle.

\section{Une ville dédoublée}

Fès est la première ville maghrébine que j'ai découverte et visitée. J'attendais beaucoup de ce séjour. Je n'ai guère été déçu. En effet ce premier contact s'enracinait dans le désir ancien de connaître une autre forme d'urbanisme que celle répandue en Europe. L'expérience humaine du voyage se décline de bien des manières : on peut se demander s'il existe un regard spécifiquement géographique que l'on peut espérer différent du regard touristique qui s'en tient à l'exceptionnel et au pittoresque, oublie ou rejette (voire nie) ce qu'il considère comme trop quotidien, prosaïque, ou « laid ». Il y a un enjeu éthique dans ces divers regards. La sélection des lieux encouragée par les guides qui, comme leur nom l'indique, signalent ce qui vaut ou non la peine d'être vu (ici : rien à voir), exerce une violence plus ou moins directe sur les lieux, finit souvent par les dégrader ou les subvertir irrémédiablement, en en déformant le sens et les usages, pour peu que les populations locales n'aient pas clairement conscience des dangers encourus.

Le tourisme est en fait une pratique paradoxale car si elle peut engendrer des espaces superlatifs (c'est-à-dire toujours " très » ou " le plus » quelque chose) à partir d'à peu près n'importe quoi, elle peut aussi détruire son objet à peine celui-ci constitué. Elle correspond, en ce cas, à une forme d'aliénation effectivement supérieure car elle joue à la fois au niveau du discours mais aussi des réalisations, des aménagements. Le vilain mot de

Mircea Eliade. René Guénon, à cause de sa position farouchement antimoderne, a suscité et suscite toujours des réactions parfois très hostiles, même s'il a été notamment lu par Antonin Artaud, Raymond Queneau ou encore Michel Leiris et André Breton. Ses adversaires ont eu beau jeu de considérer Guénon comme un penseur d'extrême droite, une lecture attentive de ses travaux invalide cette thèse. Mais ses critiques acerbes envers le monde académique ont sans doute joué un rôle non négligeable dans sa mise à l'écart. Sur René Guénon, on peut se référer au numéro du Cahier de l'Herne qui lui a été consacré en 1985.

10. Ce terme de tradition est souvent mal défini, et suscite bien des polémiques. Tradition et coutume sont souvent confondus, tout comme tradition et traditionalisme. Or, la tradition est d'abord transmission d'un savoir, de pratiques, de valeurs qui doivent être reformulées, remotivées, voire revivifiées à chaque génération pour pouvoir continuer à exercer un rôle d'encadrement mais aussi de stimulant et de référence pour une société ou un groupe donnés. Cette tradition doit surtout être appropriée pour pouvoir fonctionner. Si effectivement on ne fait que conserver, il y a risque d'étiolement, de sclérose. Guénon considérait comme « traditionnelles » les sociétés qui plaçaient le sacré au centre de leur vie. 
«touristification », par son côté un peu ridicule, n'en exprime pas moins cette espèce de transformation et de mystification qui peut parfois être sans retour. Les lieux concernés risquent d'en être dénaturés, dépossédés, et de ne plus offrir qu'une parodie amoindrie de leur réalité. La quête de $l^{\prime}$ « authentique » propre au touriste, liée à un désir de retrouvailles avec des origines souvent mythifiées, encourage à effacer ou ignorer l'actuel pour retrouver, notamment dans les paysages, une sorte de passé reconstitué dans un but essentiellement esthétique, en rapport avec les paysages imaginés par les peintres européens de notre modernité ${ }^{11}$. Écueil difficile à éviter pour une «ville d'histoire » comme Fès. Mais Fès est bien évidemment beaucoup plus qu'une ville d'histoire.

La ville se donne à voir actuellement, à l'instar de nombreuses villes du Maghreb, comme une ville dédoublée qui a connu une présence européenne suffisamment longue pour permettre aux autorités coloniales d'effectuer des aménagements conséquents. On trouve donc à l'ouest la ville ancienne (médina), et à l'est la ville nouvelle (coloniale et post-coloniale). Celles-ci sont juxtaposées et non imbriquées l'une dans l'autre (figure 1). D'autres banlieues, vraiment en périphérie, sont séparées de ces deux centres par des routes, ou tout simplement éloignées; le tout compte un peu plus d'un million d'habitants. En général, en venant de l'étranger, on découvre d'abord la ville nouvelle, puis la vieille ville, pour peu que l'on veuille $\mathrm{y}$ entrer, car celle-ci présente un aspect très compact qui peut dérouter et intimider un visiteur non averti. En effet la médina, en dehors de quelques aménagements relativement extérieurs, n'a pas fortement changé d'aspect, même si cette dernière a connu bien des crises. On a donc deux entités urbaines clairement distinctes, et même si la ville nouvelle a pris son essor depuis l'indépendance, cessant d'être seulement la ville des Européens, elle n'en conserve pas moins un caractère européen. Du reste, elle apparaît un peu moins dans les brochures touristiques et les divers guides disponibles sur Fès, comme si son caractère «typique » était insuffisant pour proposer aux touristes un quelconque dépaysement. De là à considérer que ce dédoublement puisse constituer une métaphore de la double culture urbaine du Maroc, il n'y a qu'un pas. Pourtant, il n'est pas certain que le principe du dédoublement puisse être étendu au pays tout entier.

En effet, cette juxtaposition s'accompagne aussi d'une superposition, d'un mélange plus ou moins réussi entre différents types d'urbanité, comme dans le cas de Fès Jdid (figure 2), où l'ancien quartier juif, vidé de ses habitants dans l'après-guerre, est laissé en partie à l'abandon. Cette disparité n'est pas ressentie de façon directe, mais plutôt de manière fragmentaire, du

11. Un cas d'école est celui de Gauguin. Cf. Jean-François Staszak, Géographies de Gauguin, Paris, Bréal, 2003. Il y démontre brillamment comment l'imaginaire du peintre a préparé les aménagements touristiques actuels des îles polynésiennes en accord avec la demande des touristes venus en quête des paysages des tableaux... 
fait de l'éclatement des sites et de la difficulté à se faire une représentation globale de la ville. Autant les rues droites et larges de la ville nouvelle, tout à fait en accord avec la grammaire spatiale des villes européennes modernes, se laissent appréhender sans problème, autant les ruelles souvent très étroites de la vieille ville, l'absence de perspective, bloquent cette vision d'ensemble, ou la rendent difficile.

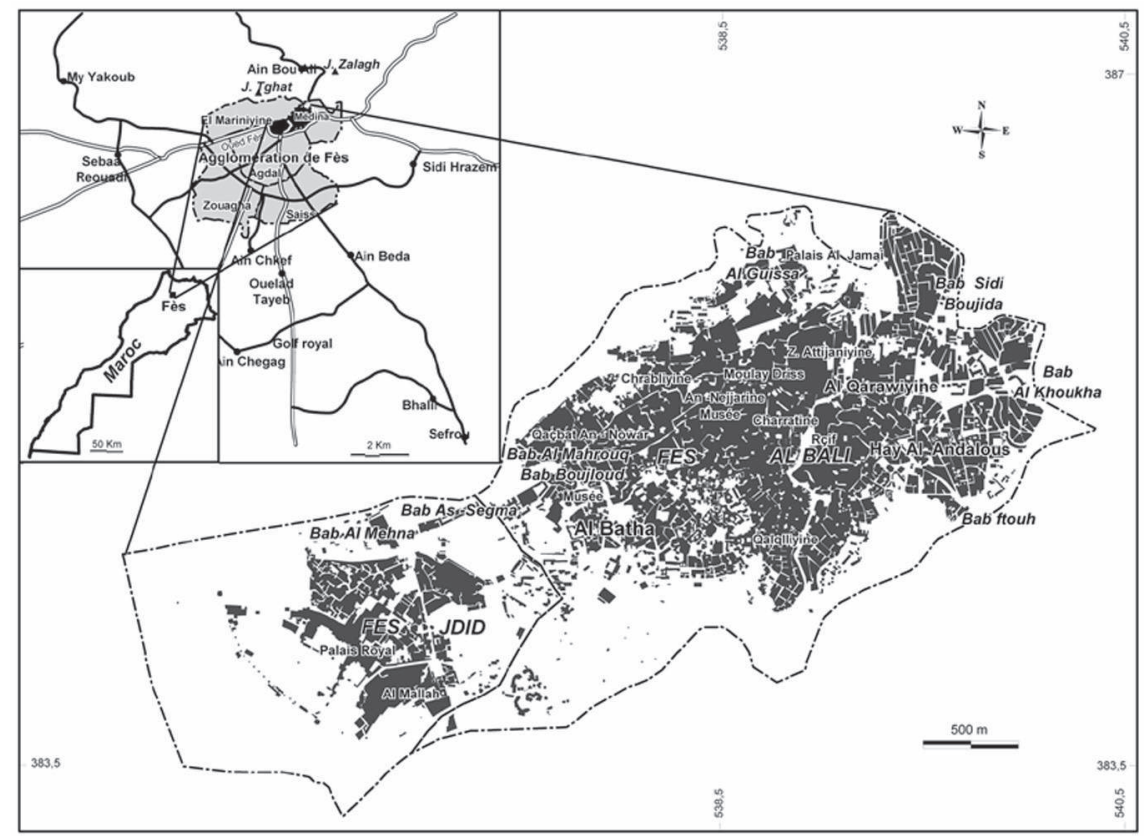

Figure 1 : Plan général de Fès, d'après Akdim et Laaouane, 2010, p. 11.

La vieille ville avec ses rues, portes et murailles évoque les villes médiévales par ses commerces regroupés par spécialité, par la quasi-absence de voitures et par le foisonnement d'activités qui se déploient à même la chaussée. Les odeurs y sont fortes, en partie à cause de la présence d'animaux de bât dans les ruelles qui représentent le seul véhicule de transport des marchandises tant les trajets sont heurtés; il s'y mêle aussi parfois des miasmes d'égout, même si les rues sont plutôt propres, et des odeurs d'épices, de parfums, de cuisine selon le quartier où vous vous trouvez (ou perdez). Il n'y a pas de noms de rue clairement indiqués. La topographie est complexe, pleine de montées et descentes, mais qui permettent toutefois de se repérer dans le lacis des ruelles. Beaucoup de murs sont aveugles puisque les maisons sont ouvertes sur les cours intérieures et non vers l'extérieur. Les nombreux minarets diffusent régulièrement les voix enregistrées des muezzins à travers la ville. D'aucuns diraient que c'est là une sorte d'archaïsme. Mais faut-il s'en arrêter là? Tenir ce discours (et l'urbanisme 
adopte souvent une posture très " progressiste » en la matière ${ }^{12}$ ), maintient une forme de colonialisme pervers, qui perpétue un jugement de valeur basé sur l'opposition binaire entre avance et retard, tradition et modernité. On classe alors une ville par rapport à une sorte de hit-parade des villes internationales et fières de l'être. Mais Fès n'est ni Paris, ni Berlin, ni Tokyo ${ }^{13} \ldots$ Elle est à la fois différente et au-delà.

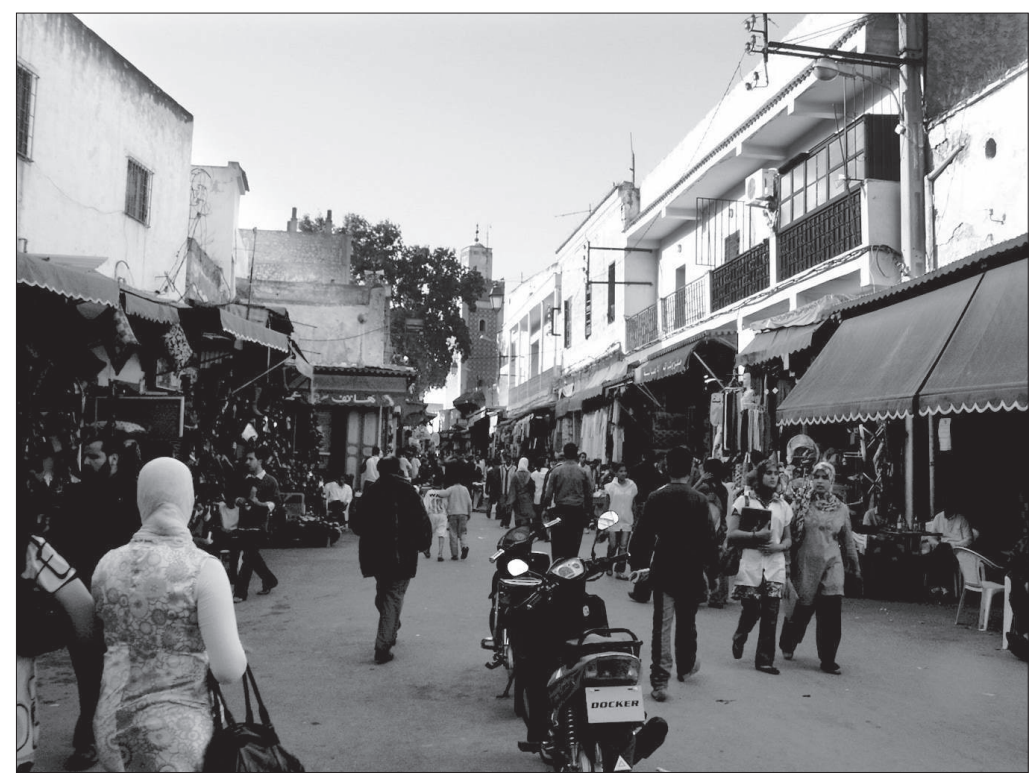

Figure 2 : Vue de Fès Jdid actuellement. Photo B. Gruet 2008.

On distingue par instants les maisons, les madaris (écoles coraniques, voir la figure 3), les mosquées, très nombreuses, et les fontaines dont la plupart ne fonctionnent plus et sont malheureusement souvent devenues des dépotoirs. Quand on sait que le système hydraulique de Fès était, à son origine, très sophistiqué, alimentant un triple réseau d'adduction d'eau, on ne peut que s'interroger sur la dégradation de la ville tout entière. On peut d'ailleurs y voir un raccourci saisissant de l'évolution récente de la

12. Un très bon exemple de cet urbanisme progressiste est celui de Sigfried Giedion, qui dans son ouvrage Espace, temps, architecture (1941), dresse un tableau quasiment évolutionniste de l'architecture et de l'urbaniste. Voir Florinel Radu, "Re-lire Giedion », EspacesTemps.net, Livres, 28 février 2005, http://www.espacestemps.net/articles/re-liregiedion/.

13. Par l'entremise de certaines universités chinoises, la mode des classements de ville a fleuri ces dernières années, en accord avec la mise en avant et la très forte médiatisation des « champions » de l'urbanisme. Pensons en particulier au chantier des Halles ou encore au projet du Grand Paris... 
ville : alors que Fès a été l'un des fleurons des villes d'Islam, elle semble se débattre à présent avec des problèmes de gestion et de fonctionnement, que le classement de l'UNESCO n'a pu sensiblement modifier. La ville apparait comme l'ombre d'elle-même. Le tourisme quant à lui incite à la parodie et fige la ville dans des clichés que les fassis semblent perpétuer avec parfois une certaine complaisance vis-à-vis de leurs visiteurs. Pourtant le passé de la ville et sa beauté restent parfaitement tangibles, mais ils sont comme recouverts. Comment accéder, dans ces conditions, aux caractéristiques essentielles de cette ville? Échappe-t-elle à la fragmentation qui semble le lot de beaucoup de villes actuelles? À ces questionnements l'œuvre de Burckhardt apporte matière à réflexion.

\section{Du motif à la ville entière : Fès, ville d'Islam de Titus Burkhardt}

Notre auteur a rédigé trois livres se faisant écho les uns aux autres: Fès, ville d'Islam, Sienne, cité de la Vierge, et Chartres et la naissance de la cathédrale. Dans chacun de ces livres, il s'est efforcé de restituer la culture spécifique de ces villes dans toutes ses dimensions, y compris en termes d'expérience spirituelle. C'est à cette lumière qu'il faut aborder Fès, ville d'Islam, livre qui utilise les différentes échelles géographiques comme autant de niveaux de signification. Pour Burkhardt, une ville comme Fès ne peut se comprendre qu'en approchant la spiritualité musulmane comme un centre autour duquel s'articulent et se structurent tous les autres discours; discours dont l'espace est à la fois la matrice, le support et le résultat. En effet, l'espace peut se prêter à une forme d'interprétation, voire d'herméneutique, et c'est ce qui en fait toute la richesse et l'aspect véritablement polyphonique.

L'auteur dresse, à travers la ville, un tableau de la société marocaine dans son ensemble, et de l'islam en général. À la lecture de ce singulier ouvrage, on voit ressortir un trait tout à fait marquant : la profonde cohérence entre les plus petits détails de la ville et la ville dans son ensemble. Cet enchaînement des différents éléments entre eux vers une forme d'unité supérieure fait exister la ville en tant que telle, mais il permet aussi d'insister sur les composantes humaines à l'origine de la ville, de sa fondation jusqu'à l'époque où Burckhardt y a vécu et étudié (figure 4).

On pourrait légitimement se demander si l'auteur ne continue pas, d'une certaine manière, ce qu'il a été convenu d'appeler l'orientalisme ${ }^{14}$. À mon sens il n'est pas possible de faire une telle confusion, sauf peut-être à cause de l'attrait premier de Burckhardt pour les arts islamiques. Mais rapidement on se rend compte à la lecture, qu'on est loin de cette fascination superficielle souvent reconnaissable dans les écrits des orientalistes. Titus Burckhardt avait une parfaite connaissance de l'arabe et avait opéré une

14. C'est-à-dire un goût pour l'« Orient », vu au travers du prisme de l'entreprise coloniale. Voir à ce propos l'article « Orientalisme» de Daniel-Henri Pageaux et Christine Peltre dans l'Encyclopcedia Universalis. 


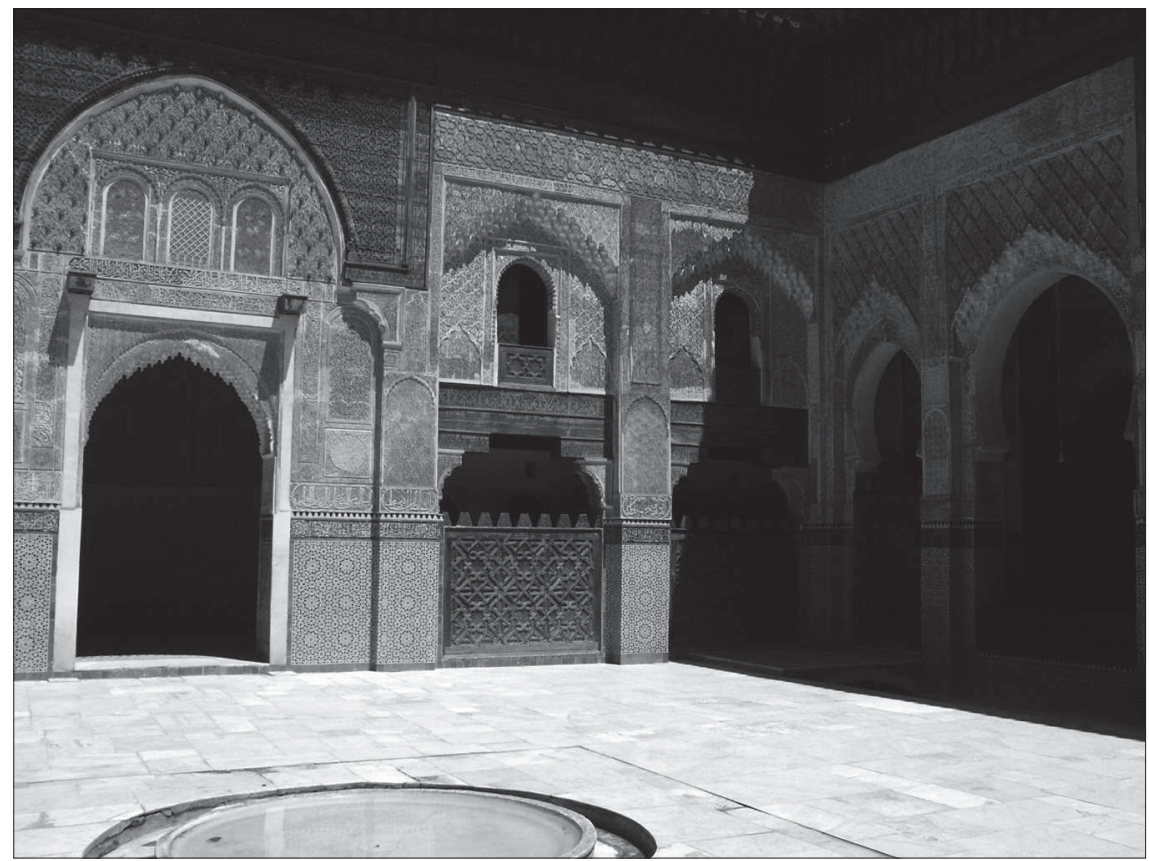

Figure 3 : Vue intérieure de l'école coranique Bou Inâniya. Photo B. Gruet 2008.

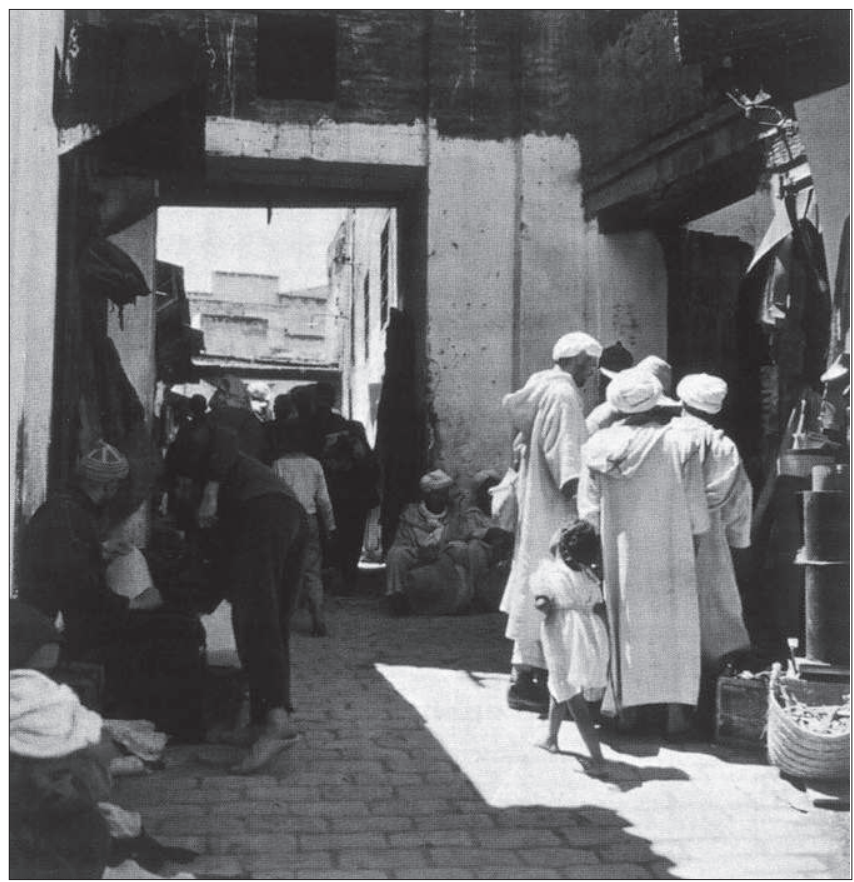

Figure 4 : Marché du cuir dans la vieille ville, d'après l'ouvrage de T. Burckhardt, p. 76. 
immersion totale dans la culture fassie. Le plan du livre est le suivant : «Fès, une ville humaine »; «Fès »; « La ville et le désert »; « Le califat »; « La ville d'Idris le saint »; « La maison »; «Le savoir traditionnel» (évocation de l'ésotérisme musulman et de sa présence dans la ville); « La chaîne d'or » (évocation du soufisme et des tariqa); "L'irruption du monde moderne ».

Tout cela forme une progression subtile et certainement nostalgique car le dernier chapitre est très critique vis-à-vis des évolutions récentes du Maroc et de la ville de Fès en particulier. Mais cette nostalgie fait partie du projet même de l'auteur de retrouver et de maintenir vivant un héritage qu'il considérait comme essentiel, au sens propre. Plus qu'un simple héritage, il s'agit d'un legs à transmettre et à faire fructifier. C'est du reste ainsi qu'il faut comprendre le mot tradition.

De la parure, du vêtement ou du décor à la forme générale de la ville, les éléments s'emboîtent et se répondent dans un rapport métonymique que rend possible la présence du sacré. Cette importance conférée au sacré peut être contestée mais force est de constater que Fès, en tant que ville musulmane, fondée au $\mathrm{IX}^{\mathrm{e}}$ siècle par Idris II, illustre les principes de l'islam ${ }^{15}$, dans la mesure où l'espace peut exprimer une dimension spirituelle partagée par l'ensemble de ses habitants, sans distinction de culture ni de richesse. À cet égard, les exemples abondent tout au long du livre. Le chapitre sur la maison est très éclairant, car on y saisit la cohérence entre les motifs « décoratifs » qui en réalité ont tous une signification symbolique très forte, et l'aménagement général des bâtiments, puis des quartiers et enfin de la ville entière (figures 5 et 6 ). Mais cette cohérence ne se retrouve pas seulement au niveau de la morphologie spatiale ou encore de la culture matérielle. On l'aperçoit aussi à travers les activités humaines, et notamment artisanales, qui apparaissent comme autant de reflets du sacré. En effet, les professions s'appuient sur des légendes qui font remonter leur origine à des ancêtres mythiques. C'est ainsi qu'un fabricant de peignes, métier humble s'il en est, se réclame de Seth, personnage de la Genèse biblique, ce qui lui permet, une fois de plus, de se rattacher à une filiation non seulement spatiale, mais historique. C'est sans doute ce caractère transhistorique qui rattrape la ville et finit par l'envelopper : la ville n'est pas que le support extérieur pour la vie des différentes communautés qui s'y sont installées, elle est aussi un creuset tant culturel que cultuel, et surtout elle évoque, dans sa topographie même, l'histoire sainte, c'est-à-dire les lieux et les personnages éminents non seulement pour la vie politique de la ville, mais aussi artisanale, économique et religieuse bien sûr.

Fès est donc à la fois une ville fondée historiquement et le reflet d'un archétype de cité céleste ou, à tout le moins, d'une cité régie par la loi 


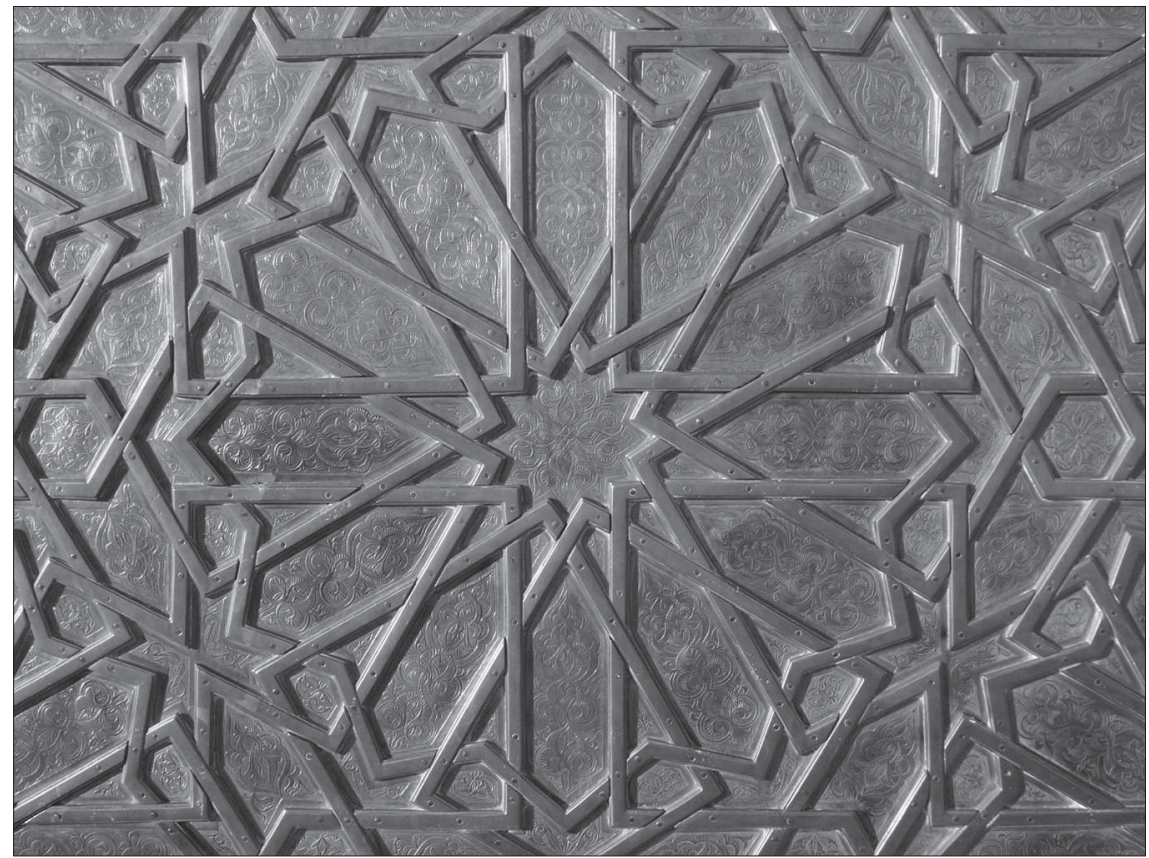

Figure 5 : Motif ornemental de la porte du palais royal de Fès, en « toile d'araignée de Dieu », allusion au « miracle de l'araignée » du Coran. Photo B. Gruet 2008.
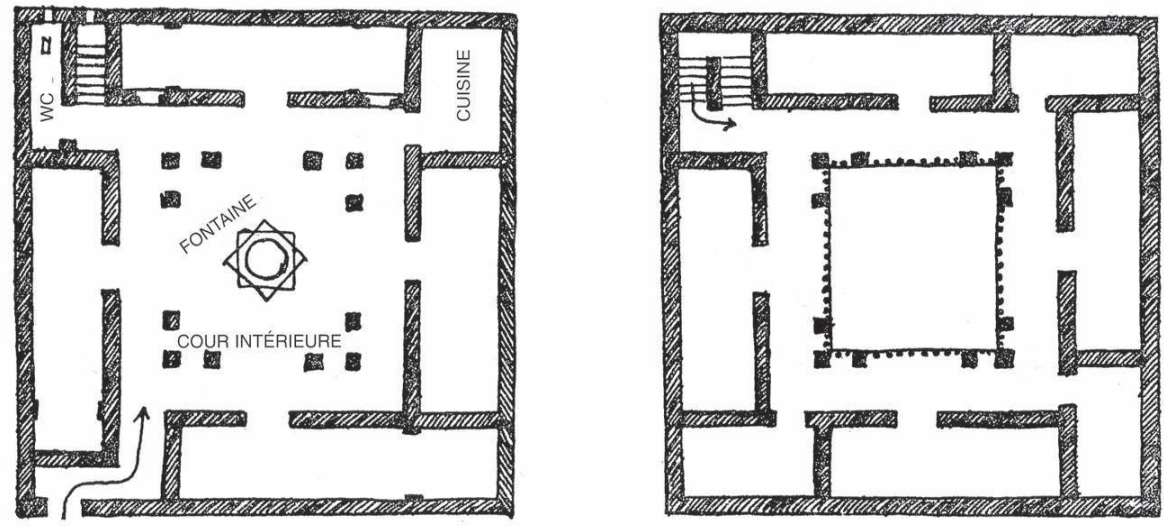

Figure 6 : Plan du rez-de-chaussée et de l'étage d'une maison traditionnelle, d'après l'ouvrage de T. Burckhardt, p. 89. 
divine, ici la loi coranique ${ }^{16}$. C'est cette loi qui, à son tour, fait le lien avec l'arrière-plan symbolique et spirituel de la ville. Arrière-plan car, par définition, il ne peut être directement aperçu, mais bien plutôt goûté, comme le disent les soufis à propos de leur expérience du divin. Or, Fès, en tant que cité sainte de l'islam (ce qu'elle est officiellement), ne saurait se priver de cette influence, ou plus exactement de cette source. De ce point de vue, l'oued Fès qui irrigue encore la ville apparait comme la métaphore du verbe divin irriguant la cité des hommes et faisant fleurir la vie. L'image est aussi simple que directement vérifiable, même si à présent, comme je le remarquais auparavant, la plupart des fontaines sont devenues des dépotoirs. C'est certainement un signe des temps, dans la mesure où l'eau ne coule plus dans la ville. Les canalisations installées récemment ont défoncé les sols fragiles, déstabilisé les maisons et les murailles, comme si cette autre logique, surajoutée à celle constitutive de la ville, ne pouvait que provoquer déséquilibres et injustices.

Par ailleurs, lorsque Burckhardt parle des « savants », et en profite pour évoquer ce qu'il nomme les sciences traditionnelles, c'est une fois de plus pour insister sur l'absence de séparation entre les activités les plus quotidiennes, voire les plus triviales, et l'accomplissement spirituel proposé à tout croyant. Bien entendu, cela s'applique à une culture encore majoritairement musulmane, mais c'est encore vérifiable car, même dans le cas de la diversification actuelle des croyances et des pratiques, la ville propose toujours un cadre pour l'épanouissement spirituel, qui peut échapper à la plupart des visiteurs étrangers. Pourtant, de proche en proche, s'opère une remontée vers un type de connaissance bien particulier, connaissance diffusée dans les écoles coraniques, mais aussi dans les tariqa, ou confréries initiatiques de la ville.

À ce titre, on peut volontiers parler de la saveur spirituelle d'un lieu, comme le font les soufis ${ }^{17}$, afin d'insister sur la charge symbolique tout à fait particulière, propre à développer et à laisser s'épanouir une certaine forme de sensibilité. La question est de savoir si cette lecture de l'espace urbain à la lumière du sacré est toujours pertinente alors que, depuis le moment où le livre a été écrit, plusieurs décennies se sont écoulées.

\section{La séduction du tourisme, l'héritage colonial et le statut de la ville actuelle}

La ville actuelle continue d'accueillir les fidèles et pèlerins, bien entendu, mais le développement de ses banlieues tout comme la pénétration des modes

16. C'est-à-dire la charia, que l'on peut traduire par «voie commune». Mais il faut rappeler qu'il existe plusieurs charias, comme il y a plusieurs écoles de droit coranique. Ceux qui brandissent « la » charia comme l'unique version du droit musulman sont donc dans l'idéologie...

17. L'expression "goût spirituel », al-dhawq, se rattache directement à la spiritualité soufie. Voir Faouzi Skali, La Voie soufie, Paris, Albin Michel, 1985. 
de vie modernes dans la vieille ville donnent une impression de dilution, voire de désagrégation de l'unité et de l'harmonie de la ville. Problèmes d'approvisionnement en eau, problèmes de circulation, problèmes de densités de population, problèmes de délabrement des édifices anciens. La liste des maux est longue. Cette situation est sensible aux abords de la vieille ville : l'espace urbain se délite et semble hésiter entre plusieurs logiques, sans oublier les effets du tourisme et de l'internationalisation de l'immobilier rendus palpables par la présence de luxueuses villas isolées en bordure de la ville ou par la pression immobilière exercée par les acheteurs occidentaux dans les secteurs anciens de la ville (figure 7). Depuis 1980, de nombreuses actions ont certes été entreprises pour remédier à ces problèmes, ou tout au moins pour les atténuer. Mais toutes ces initiatives sociales, patrimoniales, touristiques donnent l'impression d'être limitées dans leurs effets car surimposées à un espace urbain obéissant à des principes qui échappent aux politiques actuelles.

Il n'est pas question de tomber dans un discours réactionnaire ou encore de fustiger par principe le présent de la ville, mais de poser la question de l'harmonie, question qui peut sembler désuète ou naïve mais qui est en fait centrale car elle recouvre des enjeux politiques majeurs. Les thèmes liés à la « gouvernance » ou encore au " développement durable » renvoient au fond à ce problème d'harmonie. J'ai eu l'occasion de l'aborder ailleurs, je ne m'y appesantirai donc pas ici ${ }^{18}$. Mais dans tous les cas, les aspects civiques de ces problèmes méritent que l'on s'y attarde sérieusement.

Pour Burckhardt, la cause est entendue : la colonisation a apporté des ferments de déséquilibre et de « déformation » de la ville sainte. Entendonsnous bien : il ne s'agit pas de figer ou d'imaginer une essence immuable de la ville, mais bien plutôt de rendre compte d'une sorte d'acculturation marquée par le «a » privatif : perte de culture au profit d'une sorte de pastiche appauvri de ce qui pourrait être considéré comme un " modèle » européen. Burckhardt est très dur lorsqu'il aborde ce thème, et il cite en guise d'exemple une conversation rapportée par l'écrivain italien Edmondo de Amicis, en 1879. Je reprends le début de la citation :

Aujourd'hui j'eus une vive discussion avec un marchand de Fès, avec l'intention d'apprendre ce que ces « Maures » pensent de la civilisation européenne [...]. C'était un bel homme d'environ quarante ans au visage sincère et sérieux qui, dans ses voyages d'affaires, avait visité les villes les plus importantes d'Europe occidentale et qui avait séjourné longtemps à Tanger, où il avait appris un peu d'espagnol [...]. Je le questionnai sur l'impression que les grandes villes d'Europe avaient faite sur lui [...]. Il me fixa et répondit froidement: «Grandes avenues, beaux magasins, beaux palais, bons ateliers, et tout très propre. »

Par ces paroles, il semblait avoir mentionné tout ce qu'il y a de louable chez nous. « N'avez-vous rien trouvé d'autre chez nous qui soit beau et bon? »,

18. Brice Gruet, «Roma Æterna. Temps, mémoire et harmonie », Géographie et cultures, $\mathrm{n}^{\circ}$ 65, La ville se refait-elle?, 2009, p. 6-13. 
continuai-je. Il me regarda d'un air interrogateur. « Est-il donc possible, insistai-je, qu'un homme intelligent comme vous, qui a pu visiter plusieurs pays si merveilleusement supérieurs à sa propre patrie, n'en parle pas avec étonnement ou du moins avec l'émotion d'un enfant de la campagne qui a vu le palais d'un pacha? Qu'y a-t-il donc dans ce monde qui puisse provoquer votre étonnement? Quelle espèce d'homme êtes-vous? Comment vous comprendre? »- « Perdone Usted », répliqua-t-il froidement, « c'est à moi de ne pas vous comprendre. Je vous ai mentionné toutes les choses que j'estime meilleures que ce que nous avons chez nous; que dirai-je de plus? Faut-il donc que je vous dise quelque chose que je ne tiens pas pour vrai? Je répète que vos rues sont plus larges que les nôtres, que vos magasins sont plus beaux, que vous possédez des ateliers comme nous n'en possédons pas, de même des palais. Mais c'est là tout. Je n'ajouterai qu'une chose : que vous savez plus que nous, parce que vous avez plus de livres et que vous lisez beaucoup ${ }^{19}$ ».

On peut bien lire, à travers les questions de De Amicis, toute la dépréciation et la condescendance d'un Européen envers une population différente. C'est malheureusement assez banal pendant la période coloniale, mais cela n'en reste pas moins consternant. Pour Burckhardt, le rôle de la religion, à la fois sur les plans social et individuel, reste l'une des principales marques de la culture fassie, et son effacement, ou son recouvrement par d'autres usages, pose un problème difficile à dépasser. L'un des indices de ces changements relevé par notre auteur, c'est le recul progressif des habits marocains au profit des vêtements de style européen. Pour lui, ces habits traduisent un changement des plus importants.

Laissons-lui la parole :

Toutefois, la libération politique du Maroc ne mit pas fin à son asservissement spirituel. Elle l'accéléra au contraire, car pour être aujourd'hui autonome, un État doit disposer des moyens techniques inventés par l'Europe. Leur adoption entraîne la modification de toutes les formes d'existence. Or, si la forme d'existence change, son contenu spirituel se modifie également. Dans une culture authentique, marquée par la conception de Dieu, il n'existe guère de formes purement extérieures dont la perte serait sans importance. Celles qui sont transmises traditionnellement, qu'il s'agisse des lois, des coutumes et même de l'art, lequel s'appuie sur l'artisanat, sont toutes conçues pour mener de l'extérieur vers l'intérieur, vers le sens éternel de la vie. Seul celui qui a reconnu la signification des formes extérieures, le saint ou le sage, peut s'en passer : l'homme ordinaire en est incapable. La désintégration de ce mode de vie traditionnel dans le Maghreb d'aujourd'hui est plus radicale qu'elle ne le fut jamais dans l'histoire européenne; par sa soudaineté sans doute, mais surtout parce que le nouvel ordre, emprunté à un monde étranger, est sans rapport avec l'ancien. Souvent, la destruction de formes apparemment superficielles entraîne des conséquences globales ${ }^{20}$.

19. Titus Burckhardt, op. cit., p. 147.

20. Titus Burckhardt, op. cit., p. 153. 
À l'heure du Printemps arabe et de la chute des régimes autoritaires, on peut se demander dans quelle mesure ce genre de discours importe et à quoi il peut correspondre, sinon à un point de vue extrêmement rétrograde et conservateur. Pourtant, on ne peut soupçonner Burckhardt de sympathie pour l'islamisme, ni même de postmodernisme mal digéré, et encore moins de colonialisme : son texte a été écrit dans les années 1950. On est donc bien loin des colonial, ou même post-colonial studies actuelles. Alors, comment classer son point de vue, sinon comme antimoderne? Comment le comprendre?

L'idée, au fond très simple, défendue par Burckhardt, c'est que, dans une société traditionnelle, au sens où l'entend René Guénon, tout se tient, du plus petit au plus grand, et tout se rattache au sacré en tant que catégorie transcendante, de proche en proche. En ce cas, on peut considérer que l'aspect systémique de la culture revêt à la fois une certaine plasticité, une certaine capacité d'adaptation à la nouveauté, mais aussi une indéniable fragilité face à des changements trop brutaux. Et surtout, ce système englobant suppose également les valeurs, comme les fins dernières envisagées par une population donnée. Ce qui demeure, ce qui disparaît et ce qui se transforme dans ce système n'en appartient pas moins à ce que l'on pourrait appeler, comme je l'ai fait ailleurs, un éthosystème, c'est-à-dire un système qui contient non seulement la culture, mais aussi le comportement, les valeurs, et les habitus d'une population non pas refermée sur elle-même, mais en contact et en échanges constants avec d'autres groupes ${ }^{21}$. C'est la prise en considération de cet aspect systémique qui permet de rendre compte de manière mesurée et pertinente d'une ville comme Fès, qui se trouve à la confluence d'influences complexes à l'heure actuelle.

Dans un cas comme celui-ci, le clivage convenu entre tradition et modernité se rejoue de manière évidente. Si l'on ne confond pas tradition et traditionalisme, et si l'on peut, au contraire, entendre dans la tradition l'idée de filiation, il est grand temps de se pencher sur ce qui fait la sève d'un espace comme celui de Fès à la fois pour le Maroc, l'Islam mais aussi pour la ville en général. Je laisse le mot de la fin à Titus Burckhardt, qui rapporte cette citation en ouverture à son ouvrage à propos de Fès : « Voilà encore une ville humaine ${ }^{22}$. »

Brice Gruet

21. J'ai déjà abordé cette notion dans une communication restée inédite lors du congrès régional de la section «Approche culturelle» de l'Union géographique internationale à Buenos Aires, en 2007 («Aspectos culturales en las geografías económicas, sociales y políticas ») : «El enriquecimiento como aculturación. Algunas consideraciones sobre cultura, desarrollo y identidad. » Je compte y revenir dans un article spécifique, aussi me bornerai-je ici à n'en évoquer que les principaux aspects.

22. Titus Burckhardt, op. cit., p. 7. 